\title{
Comparative study of axial and femoral bone mineral density and parameters of mandibular bone quality in patients receiving dental implants
}

Published online: 15 March 2007

(C) International Osteoporosis Foundation and National Osteoporosis Foundation 2007

\begin{abstract}
Introduction: In view of the increase in the life expectancy of humans and in edentulism of the population above 50 years of age, in which the prevalence of osteoporosis is also higher, it is fundamental to better understand the effects of systemic bone mass loss on the healing process of dental implants and to determine the quality of the bone that surrounds them. The objective of the present study was to compare systemic osteoporosis (axial and femoral) and parameters of mandibular bone quality, and to evaluate osseointegration in postmenopausal women receiving dental implants. Methods: The sample consisted of 39 women aged 48-70 years, 19 with a densitometric diagnosis of osteoporosis in the lumbar spine and femoral neck and 20 controls with a normal densitometric diagnosis. Bone mineral density was measured in the patients and controls by dual-energy X-ray absorptiometry. Eighty-two osseointegrated dental implants were placed in the mandible, 39 of them in the osteoporosis group and 43 in the control group. Mandibular bone quality was evaluated by
\end{abstract}

The online version of the original article can be found at http://dx. doi.org/10.1007/s00198-006-0131-0.

Unfortunately a preliminary version of the article was published. The correct article is reproduced in full here.

M. A. L. Amorim - L. Takayama - R. M. R. Pereira

Division of Rheumatology

(Bone Mineral Metabolism Laboratory),

University of São Paulo,

São Paulo, Brazil

V. Jorgetti

Nephrology Division, School of Medicine,

University of São Paulo,

São Paulo, Brazil

R. M. R. Pereira $(\bowtie)$

Faculdade de Medicina da Universidade de São Paulo, Av. Dr. Amaldo, $455-3^{\circ}$ andar-Reumatologia, sala 3107 ,

São Paulo, SP, 01246-000, Brazil

e-mail: rosamariarp@yahoo.com

Tel.: +55-551130667213

Fax: $+55-551130667490$ classifying mandibular inferior cortical and trabecular bone on panoramic radiographs and by histomorphometric analysis of a mandibular bone biopsy. Osseointegration was analyzed after 9 months. Results: No significant difference was observed between patients with osteoporosis and controls when comparing individuals with a normal cortex and those with a severely or moderately eroded cortex determined on panoramic radiographs, although patients with MEC/SEC had lower femoral neck BMD than those with $\mathrm{NC}\left(0.688 \pm 0.17\right.$ vs. $0.814 \pm 0.144 \mathrm{~g} / \mathrm{cm}^{2}$, $P<0.012)$. Histomorphometric analysis also revealed no difference in the parameters of bone formation or resorption between the two groups. Implant failure was observed in only one case. Conclusion: We conclude that there is an association between low femoral neck BMD and poor mandibular bone quality as assessed by panoramic radiography. The loss of one implant $(1.2 \%)$ is compatible with the literature and cannot be attributed to systemic osteoporosis.

Keywords Bone histomorphometry · Bone mineral density · Dental implant · Osseointegration · Osteoporosis

\section{Introduction}

In view of the increase in the life expectancy of humans and because edentulism mainly affects the population above 50 years of age, there is a growing need for the treatment of elderly patients with dental implants. Since the prevalence of osteoporosis is also higher in this population, it is fundamental to better understand the effects of systemic osteoporosis on the healing process around dental implants and to determine the quantity and quality of the bone that surrounds them.

The changes in bone metabolism that occur in the jaw region seem to be the same as those observed in long bones [1]. The effect of a decrease in bone density on dentalimplant healing has been little investigated [2], and so far it is not known whether bone density and quality influence the favorable prognosis of dental implants. Jaffin and Berman [3] reported a higher failure rate for implants 
placed in bone with low bone mineral density (BMD) and an increase in the time of osseointegration, whereas Mori et al. [4] observed inadequate bone formation adjacent to the implant. Von Wower et al. [5] demonstrated that BMD is significantly lower in the oral cavity of patients with systemic osteoporosis than in normal subjects. In contrast, Wakley et al. [6] believe that the existence of osteoporosis in the axial or appendicular skeleton does not indicate the presence of the disease in the region of the maxilla or mandible, and if the disease is present, it does not predict an unfavorable outcome of dental implants $[7,8]$.

In view of the scarcity of studies and the controversial findings in the literature in which some authors report a relationship between osteoporosis and dental implant failure and others do not, the objective of the present study was to determine the correlation between axial and femoral osteoporosis and parameters of mandibular bone quality and to evaluate osseointegration.

\section{Subjects and methods}

Thirty-nine female patients, 19 with a densitometric diagnosis of osteoporosis in the lumbar spine and femoral neck and 20 normal controls (no densitometric diagnosis of osteoporosis and/or osteopenia) matched for age and race, were studied. The subjects were classified as osteoporotic or normal according to criteria established by the World Health Organization based on T-scores: osteoporosis, Tscore $\leq-2.5$ standard deviations; normal, T-score $\geq-1$ standard deviation [9].

BMD was measured in patients and controls by dualenergy X-ray absorptiometry (DXA) using a Hologic QDR 2000 densitometer, and is reported as $\mathrm{g} / \mathrm{cm}^{2}$. The regions analyzed were the lumbar spine (L1-L4) and femoral neck.

The patients and controls were selected from the Metabolic Bone Diseases outpatient clinic of the Rheumatology Service, University Hospital, School of Medicine, University of São Paulo. The study was approved by the Ethics Committee of the institution, and all subjects signed a free informed consent form to participate.

The general exclusion criteria were chronic diseases, current smoker, chronic alcoholism, use of glucocorticosteroids or other immunosuppressive drugs at the time of the study, and use of bisphosphonates at the time of the study or within 6 months prior to the study. Dental exclusion criteria included inability of the patients to follow instructions and perform proper oral hygiene, presence of residual roots, presence of local inflammation, diseases of the oral mucosa, a history of radiotherapy in the head and neck region, insufficient bone quantity in the region of the mandible (height $<13 \mathrm{~mm}$ and thickness $<4 \mathrm{~mm}$ ), and tooth extraction within the 4 months prior to the study [10].

\section{Dental implant}

All subjects had an indication for a dental implant and prosthetic rehabilitation in the region of the mandible.
Eighty-two osseointegrated dental implants (model Conus, INP), $4 \mathrm{~mm}$ thick and $13 \mathrm{~mm}$ long, were placed as follows: 39 implants in the osteoporosis groups and 43 in the control group.

\section{Evaluation of bone quality}

\section{Radiologic assessment of mandibular inferior cortical and trabecular bone}

The extraoral panoramic radiographs used for surgicalprosthetic planning were analyzed to evaluate the level of mandibular inferior cortical bone resorption. Radiographic reports were elaborated by three dental surgeons with experience in radiography, who were unaware to which group (osteoporosis or control) the patient belonged. The final report was made based on agreement between at least two observers.

Mandibular cortical shape on dental panoramic radiographs was determined by bilateral observation of the mandible distally from the mental foramen and was classified into three groups according the criteria of Klemetti et al. [11] and Klemetti and Kolmakow [12] as follows: (1) normal cortex: the endosteal margin of the cortex is even and sharp on both sides, (2) mildly to moderately eroded cortex: the endosteal margin shows semilunar defects (lacunar resorption) or appears to form endosteal cortical residues, (3) severely eroded cortex: the cortical layer forms heavy endosteal cortical residues and is clearly porous.

The same panoramic radiographs were also analyzed to evaluate the level of mandibular trabecular bone resorption, which was classified into two groups: dense and rarefied trabecular bone.

\section{Bone histomorphometry}

During the surgical procedure, a bone fragment was obtained from 18 subjects (9 with osteoporosis and 10 controls) and analyzed histomorphometrically to determine bone characteristics.

All histomorphometric indices are reported according to the nomenclature recommended by the American Society for Bone and Mineral Research [13]. The following histomorphometric parameters were obtained: (1) tissue area $(\mathrm{mm})$ : total area occupied by bone and medullary tissue, (2) bone area ( $\mathrm{mm})$ : area occupied by cortical and trabecular bone, (3) cortical thickness $(\mu \mathrm{m})$ : thickness of cortical bone, (4) fibrosis volume: percentage of bone marrow occupied by fibrosis, (5) osteoid surface: percentage of trabecular bone surface covered with osteoblasts, (6) eroded surface: percentage of total cancellous bone surface showing resorption cavities with or without osteoclasts, (7) trabecular number: density of the trabeculae. 


\section{Evaluation of osseointegration}

Osseointegration of the dental implants was evaluated after 9 months by clinical examination based on the criterion recommended by Smith and Zarb [14]. Periapical radiographs were analyzed according to the criteria of Spiekermann et al. [15], and the stage of peri-implantitis was evaluated as proposed by Jovanovic [16].

\section{Statistical analysis}

The distribution of each continuous variable was examined graphically and statistically for normality. Numerical data are summarized as the mean and standard deviation (SD) and percentage. Data not normally distributed were compared using the Wilcoxon nonparametric test for differences. Data normally distributed were compared using Student's $t$-test. Categorical data among groups were compared by the Fisher's exact test. Statistical significance was accepted at the $P<0.05$ level.

\section{Results}

Characteristics of the sample

The anthropometric and dental characteristics of the 39 subjects studied, including 19 with osteoporosis and 20 normals subjects, are shown in Table 1. A significantly lower weight and body mass index were observed in patients with osteoporosis compared to control. Among the 39 patients examined who received a total of 82 dental implants, 13 were totally edentulous and 26 were partially edentulous. The time between tooth loss and implant placement ranged from 230 years, with this period being longer in patients with osteoporosis than in controls $(P=0.05)$. The mean number of mandibular teeth lost was 11.47 in patients with osteoporosis and 8.45 in control subjects.
Panoramic radiography

Analysis of the morphology of the mandibular cortex on panoramic radiographs according to the classification of Klemetti and Kolmakow [12] showed a normal cortex in 5 patients with osteoporosis and in 11 control subjects, and a moderately or severely eroded cortex in 14 patients with osteoporosis and in 9 controls. There was no significant difference between the osteoporosis and control groups when comparing subjects with a normal cortex to those with severe or moderate erosion of the cortex $\left(\chi^{2} ; P=0.1053\right)$ (Table 2).

Analysis of mandibular trabecular bone on panoramic radiographs also showed no significant difference between the two groups when comparing subjects with dense and rarefied trabecular bone $\left(\chi^{2} ; P=0.3406\right)$ (Table 2$)$.

Interestingly, a significant difference was observed between panoramic mandibular cortical indices (moderately/severely eroded cortex vs normal cortex) and BMD only in femoral neck $\left(0.688 \pm 0.17\right.$ vs $0.814 \pm 0.144 \mathrm{~g} / \mathrm{cm}^{2}$, $P<0.012$ ). (Table 5).

\section{Bone histomorphometry}

Regarding bone histomorphometry, a smaller osteoid surface and greater resorption surface were observed in patients with osteoporosis, but this difference was not significant (Table 3).

Comparison of panoramic radiographs showing a moderately and/or severely eroded mandibular cortex and normal cortex with histomorphometric parameters (osteoid and resorption surface) revealed no significant difference (Table 4). Similarly, no significant difference was observed between panoramic mandibular cortical indices (severely eroded cortex vs. normal cortex) and spinal and femoral neck BMD (Table 5).

Table 1 Anthropometric and dental characteristics of patients with osteoporosis (OP) and control subjects

\begin{tabular}{llll}
\hline & OP $(n=19)$ & Control $(n=20)$ & $P$ \\
\hline Age (years) & $59.6 \pm 6.34$ & $56.9 \pm 5.25$ & $>0.05$ \\
Weight $(\mathrm{kg})$ & $55 \pm 8.66$ & $68 \pm 8.74$ & $0.0001^{*}$ \\
Height $(\mathrm{m})$ & $1.54 \pm 0.07$ & $1.55 \pm 0.05$ & $>0.05$ \\
Body mass index $\left(\mathrm{kg} / \mathrm{m}^{2}\right)$ & $23.19 \pm 3.81$ & $28.46 \pm 3.94$ & $0.0001^{*}$ \\
Race (white/nonwhite) & $17 / 02$ & $19 / 01$ & $>0.05$ \\
Time since tooth loss (years) & $13.6 \pm 8.02$ & $9.16 \pm 3.72$ & $0.05^{*}$ \\
Mandibular teeth lost $(n)$ & $11.47 \pm 5.62$ & $8.45 \pm 4.55$ & $>0.05$ \\
Totally edentulous $(n)$ & 9 & 4 & $>0.05$ \\
Partially edentulous $(n)$ & 10 & 16 & $>0.05$ \\
Implants $(n)$ & 39 & 43 & $>0.05$ \\
\hline
\end{tabular}

*Statistically significant 
Table 2 Evaluation of mandibular inferior cortical and trabecular bone on panoramic radiographs in patients with osteoporosis (OP) and controls

\begin{tabular}{lccl}
\hline Region & OP $(n=19)$ & Control $(n=20)$ & $P$ \\
\hline Cortical & & & \\
NC & 5 & 11 & $>0.05$ \\
MEC/SEC & 14 & 9 & $>0.05$ \\
Trabecular & & 13 & \\
Dense & 9 & 7 & $>0.05$ \\
Rarefied & 10 & & $>0.05$ \\
\hline
\end{tabular}

$N C$ Normal cortex, $M E C$ mildly to moderately eroded cortex, $S E C$ severely eroded cortex

\section{Evaluation of osseointegration}

With respect to osseointegration, only one patient lost the implant 5 months after placement. The characteristics of this patient were as follows: age $=67$ years, body mass index $=18.19 \mathrm{~kg} / \mathrm{m}^{2}, \mathrm{~L} 1-\mathrm{L} 4 \mathrm{~T}$-score $=-3.6$, femoral neck T-score $=-2.95$, time since tooth loss $=20$ years, classification of mandibular cortical morphology on panoramic radiographs $=$ moderately eroded cortex and rarefied trabecular bone.

Evaluation of osseointegration in the other subjects by clinical examination according to Smith and Zarb [14] showed no difference between patients with osteoporosis and controls, with all parameters being normal.

Assessment of osseointegration based on periapical radiographic findings 9 months after implant placement (5 months after loading) only demonstrated images suggestive of a reduction in the bone crest for 14 implants in seven patients with osteoporosis $(35.9 \%)$ and for 25 implants in nine control subjects $(59.5 \%)$. No peri-implant radiolucent area was observed in the other regions analyzed.

\section{Discussion}

In the present study, no significant difference was observed in the panoramic radiographic findings of cortical and trabecular bone or bone histomorphometry between patients with densitometric osteoporosis and subjects with normal densitometry, although the patients with MCE/SEC had

Table 3 Characterization of bone histomorphometric findings in 8 patients with osteoporosis (OP) and 11 controls

\begin{tabular}{llcl}
\hline & OP $(n=8)$ & Control $(n=10)$ & $P$ \\
\hline $\begin{array}{l}\text { Total area/bone area } \\
(\mathrm{mm} / \mathrm{mm})\end{array}$ & $1.46 \pm 0.40$ & $1.74 \pm 0.59$ & 0.2691 \\
Cortical thickness $(\mu \mathrm{m})$ & $77.1 \pm 44.08$ & $55.67 \pm 30.44$ & 0.2401 \\
Medullary fibrosis $(\%)$ & $4.99 \pm 9.39$ & $3.54 \pm 4.30$ & 0.8968 \\
Osteoid surface $(\%)$ & $7.55 \pm 7.37$ & $14.93 \pm 13.96$ & 0.1967 \\
Resorption surface $(\%)$ & $8.12 \pm 10.05$ & $3.23 \pm 4.89$ & 0.3599 \\
Number of trabeculae $(\mathrm{n})$ & $2.02 \pm 0.61$ & $1.92 \pm 0.58$ & 0.7270 \\
\hline
\end{tabular}

Table 4 Comparison between cortical indices on panoramic mandibular radiographs (MEC/SEC vs. NC) and histomorphometric parameters

\begin{tabular}{lcll}
\hline & MEC/SEC $(n=13)$ & NC $(n=5)$ & $P$ \\
\hline Osteoid surface (\%) & $13.80 \pm 11.83$ & $6.05 \pm 10.95$ & $>0.05$ \\
Resorption surface (\%) & $6.97 \pm 8.56$ & $1.34 \pm 3.00$ & $>0.05$ \\
\hline
\end{tabular}

$M E C$ Mildly to moderately eroded cortex, $S E C$ severely eroded cortex, $N C$ normal cortex

lower bone mineral density in femoral neck, demonstrating an association between peripheral low bone mass and mandibular cortical erosions.

Bone assessment by panoramic radiography of the mandibular inferior cortex showed no significant differences between patients with osteoporosis and controls, but moderately or severely eroded cortex was observed in $73.7 \%$ of the former compared to only $45 \%$ of controls. These data suggest involvement of mandibular cortical bone in most patients with systemic osteoporosis.

Although panoramic radiography has been used as a reference method in different studies, various investigations agree with the present results showing the lack of an association between low axial and/or peripheral BMD and mandibular bone rarefaction [11, 12, 17-26].

Mohajery and Brooks [19] conducted a study to determine whether radiographic changes could be detected in the mandible of patients with and without osteoporosis and also observed no significant difference between these two groups, concluding that it is not possible to predict systemic osteoporosis based on panoramic radiographs. On the other hand, Klemetti and Kolmakow [12] evaluated bone quality by classifying the mandibular cortex, and observed a positive relationship between low BMD and radiographically demonstrated eroded mandibular cortical bone.

Taguchi et al. [26] found a significant association between mandibular cortical erosion on panoramic radiographs and an increase in biochemical markers of bone resorption (N-terminal telopeptide of collagen), and between cortical width and spine BMD, suggesting that mandibular inferior cortical morphology might be an indicator of bone turnover and spinal BMD in postmenopausal women.

Table 5 Comparison between spinal (L1-L4) and femoral neck BMD and cortical indices on panoramic mandibular radiographs (MEC/SEC vs NC)

\begin{tabular}{llll}
\hline & $\begin{array}{l}\text { MEC/SEC } \\
(n=23)\end{array}$ & $\begin{array}{l}\text { NC } \\
(n=16)\end{array}$ & $P$ \\
\hline L1-L4 BMD $\left(\mathrm{g} / \mathrm{cm}^{2}\right)$ & $0.885 \pm 0.20$ & $0.969 \pm 0.182$ & $>0.05$ \\
L1-L4 T score & $-1.75 \pm 1.76$ & $-0.89 \pm 1.64$ & $>0.05$ \\
Femoral neck BMD $\left(\mathrm{g} / \mathrm{cm}^{2}\right)$ & $0.688 \pm 0.17$ & $0.814 \pm 0.144$ & $<0.021$ \\
Femoral neck T score & $-1.90 \pm 1.45$ & $-0.67 \pm 0.154$ & $<0.015$ \\
\hline
\end{tabular}

$B M D$, bone mineral density; $M E C$, mildly to moderately eroded cortex; $S E C$, severely eroded cortex; $N C$, normal cortex.

Data are expressed in mean $\pm \mathrm{SD}$. 
Horner and Devlin [21], studying 40 edentulous women, observed that the mandibular cortical index and subjective bone quality index were significantly correlated with mandibular cortical bone density measured by DXA [21]. However, agreement between repeated measurements of these indices was moderate to poor, particularly for the mandibular cortical index, and the authors questioned the use of these indices in clinical practice.

Other studies have demonstrated that signs of alveolar bone resorption and mandibular inferior cortical width and morphology on panoramic radiographs are associated with systemic osteoporosis [5, 27].

Similar to the findings of cortical bone assessment, in the present series analysis of mandibular trabecular bone showed no significant correlation when comparing osteoporotic patients and controls.

The assessment of mandibular trabecular bone by panoramic radiography is controversial since bone quality might be underestimated when based on radiographic density. Overlapping soft tissue, exposure time and different image receptor types are some factors that might interfere with the radiographic result [28].

In view of the controversies regarding panoramic mandibular findings, in the present study we also used histomorphometric analysis of a bone biopsy to better assess the quality and quantity of bone tissue in the mandibular region. It should be emphasized that there is no report in the Brazilian or international literature that evaluates mandibular bone quality based on histological parameters.

The studies performed by Frost [29] formed the basis for bone histomorphometry and for the understanding of the mechanisms involved in normal bone remodeling and metabolic bone diseases.

Thomsen et al. [30] developed a new and rapid method for conducting static histomorphometry on large histologic sections, and applied this method to iliac crest and lumbar vertebral bone in order to compare age-related changes at these two skeletal sites and to investigate the correlation between histomorphometric measures in these two regions. The authors concluded that static histomorphometry performed on one skeletal site does not automatically predict these measures at another site. Therefore, it is recommended that static histomorphometry be performed at the skeletal site of interest, if possible.

Based on the concepts outlined above, in the present study histomorphometric examination was performed at the site of interest. Statistical analysis of these measures also demonstrated no difference between patients with osteoporosis and controls. We also observed no association of eroded and normal cortical bone on panoramic radiographs with the histomorphometric findings despite a tendency toward a greater osteoid surface and resorption area in patients with moderate or severe cortical erosion. Thus, further studies possibly involving a larger sample size are necessary to confirm these findings.

Clinical anamnesis demonstrated a longer mean time since tooth loss in patients with osteoporosis compared to controls. These data agree with the literature showing a positive association between osteoporosis, tooth loss and edentulism [31]. In this respect, evidence indicates that treatments designed to increase BMD such as hormone replacement and bisphosphonate therapy may be associated with less tooth loss [32].

Taguchi et al. [33], evaluating the relationship among L1-L4 vertebral bone mass, tooth loss and mandibular bone mass in Japanese women, suggested that bone loss in the region of the posterior teeth is associated not only with a reduction in alveolar bone height but also in BMD. These authors concluded that alveolar bone height might be related to a decrease in lumbar spine BMD.

With respect to osseointegration, in the present study 81 of the 82 dental implants were clinically immobile after load placement. The patients did not complain of pain or neuropathy, and there were no signs of inflammation or infection in the peri-implant region.

Periapical radiographic assessment of osseointegration revealed no peri-implant radiolucent evidence suggestive of implant loss, although a reduction in the bone crest was observed that was more frequent in control subjects than in patients with osteoporosis (59.5 vs, 35.9\%). These contradictory findings possibly have no meaning since isolated assessment of the bone crest does not seem to be important for the outcome of osseointegration [15]. In addition, a crestal bone loss of 1.5-2.0 $\mathrm{mm}$ is expected in the first year of an implant [34]. Adell et al. [35] reported that changes in marginal bone height predominantly occur during the healing and remodeling phases from the installation of the implant to the end of the first year after prosthesis placement, with a mean bone loss of approximately $1.2 \mathrm{~mm}$ during these phases and subsequent annual losses of $0.1 \mathrm{~mm}$.

No radiolucent images were observed in the other implant areas analyzed radiographically in the present study, in agreement with Adell et al. [35], Branemark et al. [36], and Strid [37], who stated that a clinically stable implant is radiologically characterized by a normal bone image in intimate contact with the metal surface at the level of radiographic resolution, whereas the presence of a periimplant radiolucent image is indicative of the presence of soft tissue and consequent implant mobility.

In the present series, one implant was lost 9 months after surgery, with an osseointegration success rate of $98.8 \%$, a value compatible with studies conducted on the general population. For example, the survey by Jemt et al. [38] showed a success rate for dental implants of $99 \%$. Adell et al. [39] reviewed the survival rates of prostheses and fixtures in 759 jaws among a total of 4,636 implants and observed rates of $91-99 \% 5$ years after loading.

Although we were unable to demonstrate a significant association between systemic osteoporosis and poor bone quality evaluated by panoramic radiography and bone histomorphometry, it is interesting to note that with respect to osseointegration the only implant lost belonged to a patient with important systemic osteoporosis, and we therefore question the importance of bone health as an indicator of dental implant outcome.

Baxter and Fattore [40] reported that osteoporosis is a systemic condition with can potentially interfere with implant treatment and suggested that the maxilla and 
mandible are affected by this process. According to these authors, the prognosis of osseointegration in patients with osteoporosis might be improved if they receive medical treatment. However, there is no scientific evidence to contraindicate the use of dental implants in these patients since the bone healing process seems to be similar to that of healthy subjects.

The literature regarding this subject is highly controversial. Dao et al. [41] reported that the success of osseointegration mainly depends on the site where the implant will be installed and they do not consider systemic osteoporosis an important indicator of dental implant outcome. According to these authors, a decrease in bone mass and the presence of fracture at a site other than the mandible does not contraindicate implant placement, with the evaluation of bone quality at the surgical site being more important [41, 42].

Similarly, Zarb and Schmitt [43], who determined whether a decrease in systemic bone mass might affect the maxilla and mandible impairing the process of oral rehabilitation, concluded that, although osteoporosis results in a decrease in bone mass, especially in long bones, adequate repair and healing seem to occur. The authors suggested that advanced age and osteoporosis are not contraindications for osseointegrated implant therapy.

In summary, the present findings show that low BMD in femoral neck is associated with cortical mandibular erosions, although systemic osteoporosis is not a contraindication for dental implants. In this respect, further epidemiological studies including a larger sample and a longer follow-up period of patients receiving dental implants are necessary to reach more precise conclusions.

Acknowledgements We thank Dr. Emiko Saito Arita, Dr. Jose Tadeu de Siqueira and Dr. Terezinha Amaral for panoramic radiographic analysis and suggestions.

\section{References}

1. Herickson PA, Wallenluis K, Astrand K (1984) The mandibula and osteoporosis. Method for determining mineral content of the mandibula and radius. J Oral Rehabil 1:75-84

2. August M, Kaban LB (1999) The aging maxillofacial skeleton. In: Rosen CJ, Glowacki J, Bigezikian JP (eds) The aging skeleton. Academic Press, San Diego, pp 359-371

3. Jaffin RA, Berman CL (1991) The excessive loss of Branemark fixture in type IV bone: a 5-year analysis. J Periodontol 62:2-4

4. Mori H, Manabe M, Kurachi Y, Naguno M(1997) Osseointegration of dental implants in rabbit bone with low mineral density. J Oral Maxill Surg 55:351-361

5. Von Worwen N, Klausen B, Kollerup G (1994) Osteoporosis: a risk factor in periodontal disease. J Periodontol 65:1134-1138

6. Wakley GK, Baylink DJ (1988) Systemic influences on the bone response to dental and orthopedic implants. J Oral Implantol 14:285-311

7. Friberg B, Ekestube A, Mellstron D, Sennerby L (2001) Branemark implants and osteoporosis: a clinical exploratory study. Clin Implant Dent Relat Res 3:50-56

8. Cho P, Schneider GB, Krisan K, Keler JC (2004) Examination of the bone implant interface in experimentally induced osteoporotic bone. Implant Dent 13:79-87
9. Kanis JA, Melton LJ, Christiansen C, Johnston CC, Khaltaev N (1994) The diagnosis of osteoporosis. J Bone Miner Res 8:1137-1141

10. Weber HP, Fiorellini JP, Buser DA (1997) Hard-tissue augmentation for the placement of anterior dental implants. Compend Contin Educ Dent 18:779-784

11. Klemetti E, Kolmakow S, Kroger H (1994) Pantomograph in assessment of the osteoporosis risk group. Scand J Dent 102:68-72

12. Klemetti E, Kolmakow S (1997) Morphology of the mandibular cortex on panoramic radiographs as an indicator of bone quality. Dentomaxillofac Radiol 26:22-25

13. Parfitt AM, Drezner MK, Glorieux FH, Kanis JÁ, Malluche H, Meunie PJ, Ott SM, Recker RR (1987) Bone histomorphometry: standardization of nomenclature, symbols, and units. J Bone Mineral Res 2:595-610

14. Smith DE, Zarb GA (1989) Criteria for success of osseointegrated endosseous implants. J Prosth Dent 62:567-572

15. Spiekermann H (2000) Implantologia. Translation by Sergio Lian Branco Martins. Artmed, Porto Alegre

16. Jovanovic SA (1990) Paradontale aspecten von tandheelkundige implantaten. Tandarstprakti J K 3:16

17. Kribbs PJ, Chesnut CH 3rd, Ott SM, Kilcoyne RF (1989) Relationships between mandibular and skeletal bone in osteoporotic population. J Prosthet Dent 62:703-707

18. Kribbs PJ, Chesnut CH 3rd, Ott SM, Kilcoyne RF (1990) Relationships between mandibular and skeletal bone in a population of normal women. J Prosthet Dent 63:86-89

19. Mohajery M, Brooks SL (1992) Oral radiographs in the detection of early signs of osteoporosis. Oral Surg Oral Med Pathol 73:112-117

20. Taguchi A, Tanimoto K, Suei Y, Otani K, Wada T (1996) Relationship between the mandibular and lumbar vertebral bone mineral density at different postmenopausal stages. Dentomaxillofac Radiol 25:130-135

21. Horner K, Delvin H (1998) The relationship between two indices of mandibular bone mineral density measured by dual energy Xray absorptiometry. Dentomaxillofac Radiol 27:17-21

22. Jowitt N, MacFarlane T, Devilin H, Klemetti E, Jorner K (1999) The reproducibility of the mandibular cortex index. Dentomaxillofac Radiol 28:141-144

23. Francischone P, Tavano O (2000) Avaliação da perda óssea maxilar pela análise da radiografia panorâmica comparando com a densitometria óssea mandibular e femoral. BCI 7:24-28

24. Bulgarelli AF (2002) Osteoporose: um foco de estudo na odontologia. RPG Rev Pos-Grad 9:379-382

25. White SC (2002) Oral radiographic predictors of osteoporosis. Dentomaxillofac Radiol 31:84-92

26. Taguchi A, Sanada M, Krall E, Nakamoto T,Ohtsuka M, Suei Y, Tanimoto K, Kodama I, Tsuda M, Ohama K (2003) Relationship between dental panoramic radiographic findings and biochemical markers of bone turnover. J Bone Miner Res 18:1689-1695

27. Taguchi A, Tanimoto K, Suei Y, Otani K, Wada T (1995) Oral signs as indicators of possible osteoporosis in elderly women. Oral Surg Oral Med Pathol 80:612-616

28. Klemetti E, Kolmakow S, Heiskanen P, Vainio P, Lassila V (1993) Panoramic mandibular index and bone mineral densities in postmenopausal women. Oral Surg Oral Med Pathol 75:774-779

29. Frost HM (1964) Bone biodynamics. Little Brown, Boston, pp 315-333

30. Thomsen JS, Ebbesen EN, Moseklde L (2002) Static histomorphometry of human iliac crest and vertebral trabecular bone: a comparative study. Bone 30:267-274

31. Mohammad AR, Jones JD, Brunsvold MA (1994) Osteoporosis and periodontal disease: a review. CDA J CA Dent Assoc 22:69-75

32. Jeffcoat MK (1998) Osteoporosis: a possible modifying factor in oral bone loss. Ann Periodontol 3:312-321 
33. Taguchi A, Tanimoto K, Suei Y, Ohtsuka M, Hollender LG (1999) Relationship between bone mineral density and tooth loss in elderly Japanese women. Dentomaxillofac Radiol 28:219-223

34. Albreketsson T, Zarb GA (1989) The Branemark osseointegrated implant. Quintessence, Chicago, 262pp

35. Adell R, Lekholm U, Rockler B, Branemark PI (1981) A 15year study of osseointegrated implants in the treatment of the edentulous jaw. Int J Oral Surg 10:387-416

36. Branemark P-I, Hansson BO, Adell R, Breine U, Lindström MJ, Hallén O, Ohman A (1977) Osseointegrated implants in the treatment of the edentulous jaw. Experience from a 10-year period. Scand J Plast Reconstr Surg 16:1-132

37. Strid KG (1985) Radiographic procedures. In: Branemark PI, Zarb GA, Albrektsson T (eds) Tissue- integrated prosthesis. Osseointegration in clinical dentistry. Quintessence, Chicago, pp 317-337
38. Jemt T, Lekholm U, Adell R (1989) Osseointegrated implants in the treatment of partially edentulous patients: a preliminary study on 876 consecutively placed fixture. Int J Oral Maxillofac Implants 4:211-217

39. Adell R, Eriksson B, Lekholm U, Branemark P-I, Jemt T (1990) Long-term follow-up study of osseointegrated implants in the treatment of totally edentulous jaws. Int $\mathrm{J}$ Oral Maxillofac Implants 5:347-359

40. Baxter JC, Fattore L (1993) Osteoporosis and osseointegration of implants. J Prosthodont 2:120-125

41. Dao TTT, Anderson JD, Zarb G (1993) Is osteoporosis a risk factor for osseointegration of dental implants? Int J Oral Maxillofac Implants 8:137-144

42. Köndell PA, Nodenran A, Landt H (1988) Titanium implants in the treatment of edentulousness: influence of patients' age on prognosis. Gerodontics 4:280

43. Zarb GA, Schmitt A (1994) Osseointegration for elderly patients: the Toronto study. J Prosthet Dent 72:559-568 